\title{
Cystic ovarian teratoma as a novel tumor and growth hormone deficiency as a new condition presenting in Multiple Endocrine Neoplasia type 2B: Case reports and review of the literature
}

\author{
Renata Pomahacova a , Petra Paterovaa , Eva Nykodymova a , Eliska Vaclavikovab, Pavla Sykorovac, Katerina Personovac, \\ Ramir Katra ${ }^{\mathrm{d}}$, Ivan Subrt ${ }^{\mathrm{e}}$, Josef Sykora ${ }^{\mathrm{a}}$
}

\begin{abstract}
Background. We describe early and typical nonendocrine symptoms of Multiple Endocrine Neoplasia type 2B (MEN2B) presented in our patients with de novo M918T mutation in the RET proto-oncogene in early childhood, however, the diagnosis of MEN2B and medullary thyroid carcinoma (MTC) was confirmed late, in the second decade of life. In this paper, we emphasize the possibility of growth retardation, growth hormone $(\mathrm{GH})$ deficiency and ovarian teratoma as a new symptom of MEN2B.

Case Reports. Advanced MTC with palpable mass on the neck and nonendocrine symptoms such as marfanoid habitus, thickened lips, mucosal neuromas led to the diagnosis in case 1 at the age of 13 years and GH deficiency and nonendocrine symptoms in case 2 at the age of 11 years. The earliest feature of MEN2B was alacrima and constipation. Patient 1 was operated on for a slipped femoral capital epiphysis and for a cystic ovarian teratoma.

Conclusions. Improved awareness of nonendocrine signs of MEN2B could lead to earlier diagnosis, when surgical cure of MTC is possible. Alacrima is the first sign of MEN2B. We confirmed the possibility of growth retardation and GH deficiency in MEN2B, which had been previously rarely described. We suggest that patients with MEN2B may develop cystic ovarian teratoma, to the best of our knowledge, which has never been described so far in the literature. The results of this study could be used to guide further diagnosing of MENB2 at the early stage for better clinical outcome. We emphasize that MEN2B carries a risk for development of cystic ovarian teratoma as a novel tumor in this disease.
\end{abstract}

Key words: multiple endocrine neoplasia type 2B, medullary thyroid carcinoma, growth hormone deficiency, ovarian teratoma

Received: June 7, 2021; Revised: August 10, 2021; Accepted: August 11, 2021; Available online: August 24, 2021

https://doi.org/10.5507/bp.2021.051

(c) 2022 The Authors; https://creativecommons.org/licenses/by/4.0/

aDepartment of Paediatrics, Charles University in Prague, Faculty of Medicine in Pilsen, Czech Republic

${ }^{b}$ Department of Molecular Endocrinology, Institute of Endocrinology, Prague, Czech Republic

'Department of Nuclear Medicine and Endocrinology, 2nd Faculty of Medicine, Charles University in Prague and Motol University Hospital, Prague, Czech Republic

${ }^{d}$ Department of Otorhinolaryngology and Head and Neck Surgery, 1st Faculty of Medicine, Charles University in Prague and Motol University Hospital, Prague, Czech Republic

eInstitute of Medical Genetics, Charles University in Prague, Faculty of Medicine in Pilsen, Czech Republic

Corresponding author: Renata Pomahacova, e-mail: pomahacova@fnplzen.cz

\section{INTRODUCTION}

The hereditary form of medullary thyroid carcinoma (MTC) may occur isolated as a familial MTC or as a part of Multiple Endocrine Neoplasia type 2A (MEN2A MTC, pheochromocytoma, primary hyperparathyroidism, Hirschsprung disease) and type 2B (MEN2B). MEN2B is a very rare, but often fatal, autosomal dominant hereditary cancer syndrome, caused by activating germline mutations in the RET proto-oncogene. MTC in MEN2B is more aggressive than in MEN2A. More than 95\% of patients with MEN2B have M918T mutation in exon 16 of the RET proto-oncogene and a further $2-3 \%$ have $\mathrm{A} 883 \mathrm{~F}$ mutation ${ }^{1-4}$. The prevalence of MEN2B (0.9-1.65 per million) and the incidence (1.4-2.6 per million live births per year) are very 1 low $^{5}$. MEN2B is mainly characterized by very early onset MTC in $100 \%$ of cases, pheochromocytoma in
$50 \%$ of cases, and typical nonendocrine features including marfanoid habitus and other skeletal abnormalities (scoliosis, lordosis, kyphosis, pes cavus, pectus excavatum, high-arched palate, slipped femoral capital epiphysis), mucosal neuromas affecting the tongue, lips, buccal mucose, conjunctiva; alacrima and wide-spread ganglioneuromatosis of the gastrointestinal tract (GI) leading to GI abnormalities related to altered motility like constipation and/or diarrhea ${ }^{6,7}$. Ophthalmological signs include ptosis and everted upper eyelids. Short stature with growth below the third percentile was rarely described in children with MEN2B $\left(\right.$ ref. $^{8}$ ). Delayed puberty is reported in $40 \%$ of cases ${ }^{9}$. Ovarian carcinoid tumors may rarely develop in patients affected by MEN syndromes ${ }^{10}$. Because late diagnosis of MTC is potentionally fatal, the current guidelines of the American Thyroid Association (ATA) recommend prophylactic thyroidectomy before the age of 1 year, even 

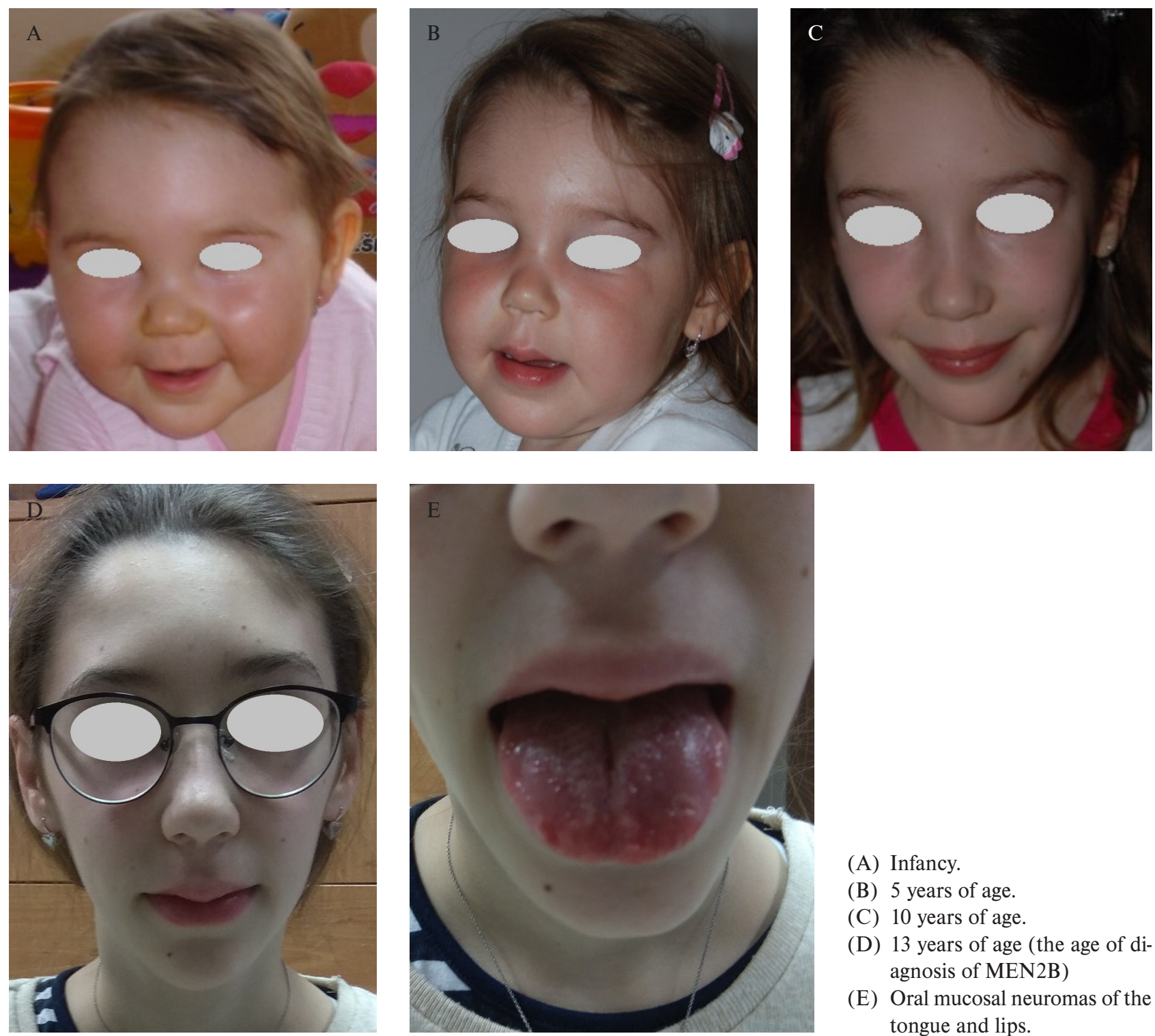
(A) Infancy.
(B) 5 years of age.
(C) 10 years of age.
(D) 13 years of age (the age of di- agnosis of MEN2B)
(E) Oral mucosal neuromas of the tongue and lips.

Fig. 1. Patient 1. The development of pathognomic clinical phenotype of MEN2B during the childhood with a thin, elongated face and enlarged lips visible from 10 years of age (C,D)

in the first months, in patients with known mutation ${ }^{11}$. Howewer, as $90 \%$ of patients carry de novo mutations, the diagnosis of MEN2B is usually late in childhood or adolescence, when MTC has already developed and even metastasized $^{7,11}$. The average age of the MEN2B diagnosis is 14.2 years $^{12}$. MEN2B presents with nonendocrine features early in childhood but often is undiagnosed for years. If affected children are identified before development of advanced malignant disease, surgical cure is possible $^{13}$. Awareness of the early nonendocrine signs of MEN2B would help to lower the age of diagnosis and improve long-term outcomes in these patients?

We report the new clinical features and outcomes of two children with MEN2B reported from the Czech Republic. Very few occurrences have been reported in the literature but none of the cases of this extremely rare disease presented with cystic ovarian teratoma. To our knowledge, we describe the first known mature cystic ovarian teratoma as a novel tumor in MEN2B. We emphasize the presence of alacrima as the first sign of MEN2B, the possibility of growth retardation and growth hormone $(\mathrm{GH})$ deficiency in children with MEN2B, less described in the literature, thus open questions still exist. The initial and typical nonendocrine symptoms of MEN2B were present in our patients with de novo M918T mutation in the RET proto-oncogene in early childhood, but the diagnosis of MEN2B and MTC was confirmed late, in the second decade of life.

\section{CASE REPORT 1}

The index patient (P1) is now a 14 year old girl. She is the first child of non-consanguineous parents of Czech descent. She was born at full term after an uncomplicated pregnancy and delivery with the birth weight $2500 \mathrm{~g}$ and the length $48 \mathrm{~cm}$. 

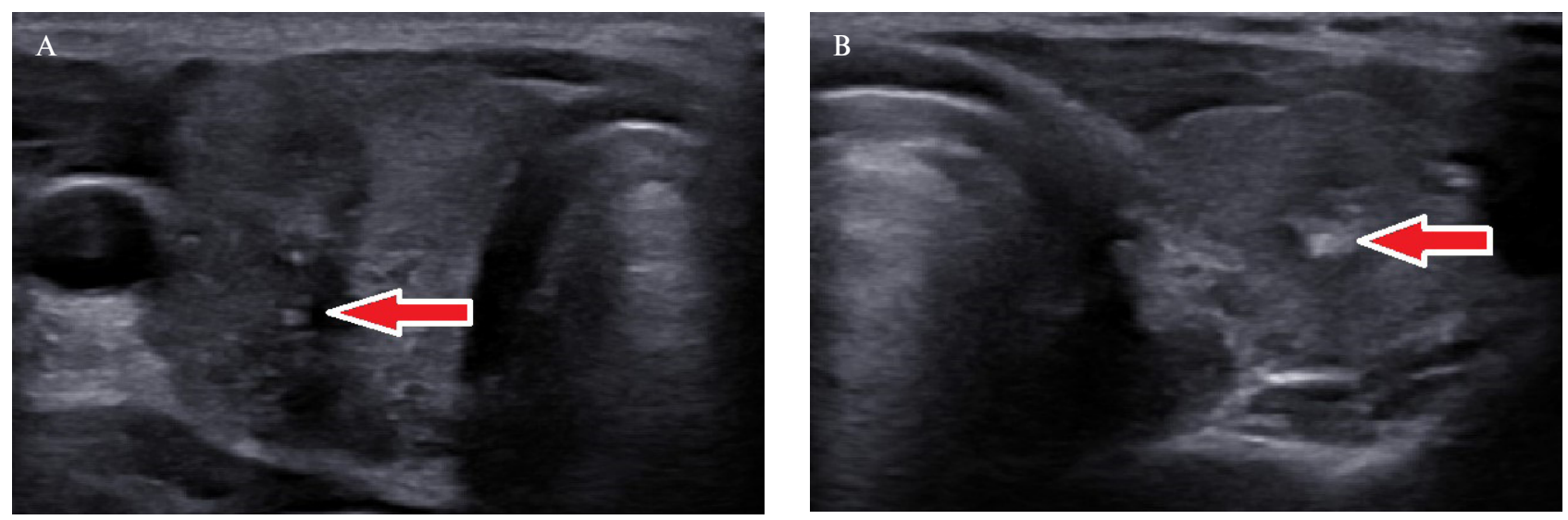

Fig. 2. Patient 1. Ultrasonography of the thyroid gland; advanced medullary thyroid carcinoma in each lobe of the thyroid gland with calcifications $(A, B)$.

In her medical history, she had failure to thrive with feeding difficulties, chronic constipation of unknown etiology since infancy and alacrima from birth. Upper gastrointestinal symptoms such as difficulty swallowing, heartburn, nausea and vomiting during childhood suggested esophageal abnormalities. She suffered from muscle hypotonia, pes cavus causing frequent falls as a toddler and tooth malposition with central diastema. She was underweight and her growth was under the third percentile till 7 years of age. Her mental development was normal. She had never been seriously ill.

At the age of 13 years, she was operated on for a slipped femoral capital epiphysis on the right side after a minor injury. At the same age, she was referred to our Outpatient Department of Endocrinology for a nodes in the thyroid gland.

On the examination, she was underweight, her BMI (body mass index) was $13.7 \mathrm{~kg} / \mathrm{m}^{2}$ (under the third percetile), her height $139 \mathrm{~cm}$ (the 25th percentile) and her puberty was advanced (Tanner stage III). She had a thin elongated face, high-arched palate, bumpy lips, mucosal neuromas of the tongue, lips, gingival hyperplasia and long, thin extremities, pes cavus and long first toe (Fig. 1). Enlarged right lobe of the thyroid gland was palpable. Her blood pressure was normal.

Ultrasonography revealed two masses in each lobe of the thyroid gland with calcifications and multiple suspected metastatic cervical lymph nodes (Fig. 2). The size of the right mass was $25 \times 15 \mathrm{~mm}$ and the size of the left mass was $5 \times 5 \mathrm{~mm}$. FNAB (fine needle aspiration biopsy) of the right mass was suspected of MTC (Bethesda classification for thyroid cytopathology). This suspicion of MTC was confirmed by high level of calcitonin 369.5 $\mathrm{pg} / \mathrm{mL}$ (normal range: $0-8$ ). Thyroid function at the time of MTC diagnosis was normal and thyroid peroxidase and thyroglobulin antibodies as a marker of autoimmune thyroiditis were negative. She underwent total thyroidectomy and cervical lymph node dissection. The surgery was difficult due to the infiltrative growth of carcinoma outside the thyroid gland (oesophagus, trachea). Histological examination confirmed bilateral MTC with metastasis in the lymph nodes.
The postoperative level of calcitonin was high $(295.3 \mathrm{pg} / \mathrm{mL}) 6$ months after the operation and 2 months after external actinotherapy. PET/CT (positron emission tomography-computed tomography) revealed suspected mediastinal metastases and a large cystic mass originating from the right ovary was found in the pelvis. The histologically cystic ovarian teratoma was removed using the laparoscopic technique. Our patient is postoperatively substituted with L-thyroxine $125 \mathrm{ug} / \mathrm{d}$, vitamin $\mathrm{D}$ and calcium due to postoperative permanent hypoparathyroidism. Her prognosis is uncertain.

Genetic analysis confirmed mutation in the RET proto-oncogene (c.2753T >C, p.M918T) in exon 16 (Institute of Endocrinology, Prague, Czech Republic). The genetic testing excluded the same mutation in her parents and in her brother. The final diagnosis of our patient was multiple endocrine neoplasia type 2B (MEN2B), de novo mutation in the RET proto-oncogene. Screening for pheochromocytoma has been negative so far (metanephrines and normetanephrines).

\section{CASE REPORT 2}

The index patient (P2) is now a 14 year old boy. He is the second child of non-consanguineous parents of Czech descent. He was born at full term after an uncomplicated pregnancy and forceps delivery with the birth weight $3340 \mathrm{~g}$ and the length $48 \mathrm{~cm}$.

He had alacrima from birth, but he didn't suffer from constipation. His psychomotor development was normal. He required dental care for tooth malposition with central diastema. He had never been seriously ill.

At the age of 10 years, he was referred to our Outpatient Department of Endocrinology for short stature. The growth was linear below the 3rd percentile without any progressive growth delay, but outside the genetically predicted range related to the parents' heights (the father's height $191 \mathrm{~cm}$, the mother's height $171 \mathrm{~cm}$ ).

On our first examination, he was underweight, his BMI was $14.5 \mathrm{~kg} / \mathrm{m}^{2}$ (10th percetile), his height $129 \mathrm{~cm}$ (under the 3 rd percentile) and no puberty was observed 

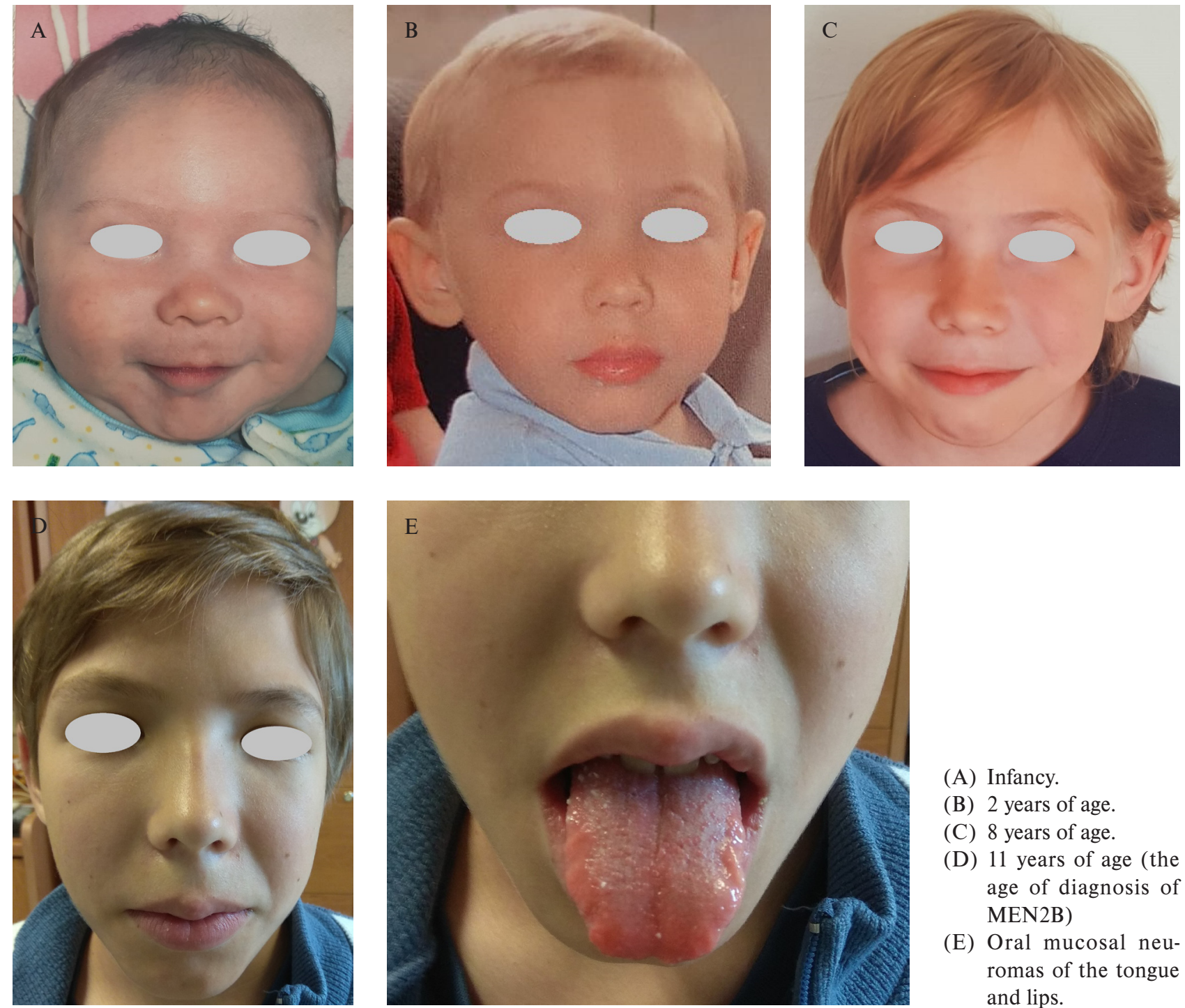

(A) Infancy.

(B) 2 years of age.

(C) 8 years of age.

(D) 11 years of age (the age of diagnosis of MEN2B)

(E) Oral mucosal neuromas of the tongue and lips.

Fig. 3. Patient 2. The development of pathognomic clinical phenotype of MEN2B during the childhood with a thin, elongated face from 10-11 years of age (D), enlarged lips were visible earlier (B,C).

(Tanner stage I). He had a proportional stature with long and thin extremities, facial stigmatization with a thin elongated face, high-arched palate, thickened lips, mucosal neuromas of the tongue and lips, low-set ears (Fig. 3).

Hormonal findings and MRI (magnetic resonance imaging) scan of the pituitary and hypothalamus confirmed idiopathic, isolated, partial GH deficiency. The maximum serum GH level during the GH stimulation tests was 17 $\mathrm{mIU} / \mathrm{L}$ (required level of $\mathrm{GH}$ is above $20 \mathrm{mIU} / \mathrm{L}$ ) and serum IGF-I (insulin-like growth factor-I) as a marker of $\mathrm{GH}$ secretion was $69 \mathrm{ng} / \mathrm{mL}$ (-2 SD). The bone age was 1 year delayed. GH therapy increased his hight velocity.

The patient's phenotype and our experience with the previous patient led us to suspicion of MEN2B syndrome at the age of 11 years. We repeatedly confirmed high level of calcitonin $97.6 \mathrm{pg} / \mathrm{mL}-78.2 \mathrm{pg} / \mathrm{mL}$ (normal range: 0-21). Ultrasonography revealed hypoechoic $5 \mathrm{~mm}$ thyroid nodule in each lobe without calcifications and without suspected cervical metastatic lymph nodes (Fig. 4). Thyroid function tests were normal.
Genetic analysis confirmed mutation in the RET proto-oncogene (c.2753T>C, p.M918T) in exon 16 (Institute of Endocrinology, Prague, Czech Republic). The genetic testing excluded the same mutation in his parents and in his sister. The final diagnosis of our patient was multiple endocrine neoplasia type $2 \mathrm{~B}$, de novo mutation in the RET proto-oncogene.

He underwent total thyroidectomy and cervical lymph node dissection. Histological examination confirmed bilateral MTC, without metastasis in the lymph nodes.

His level of calcitonin was normal $(7.7 \mathrm{pg} / \mathrm{mL}) 3$ months after the operation, indicating that the operation was curative and the prognosis is favorable. Screening for pheochromocytoma has been negative. 

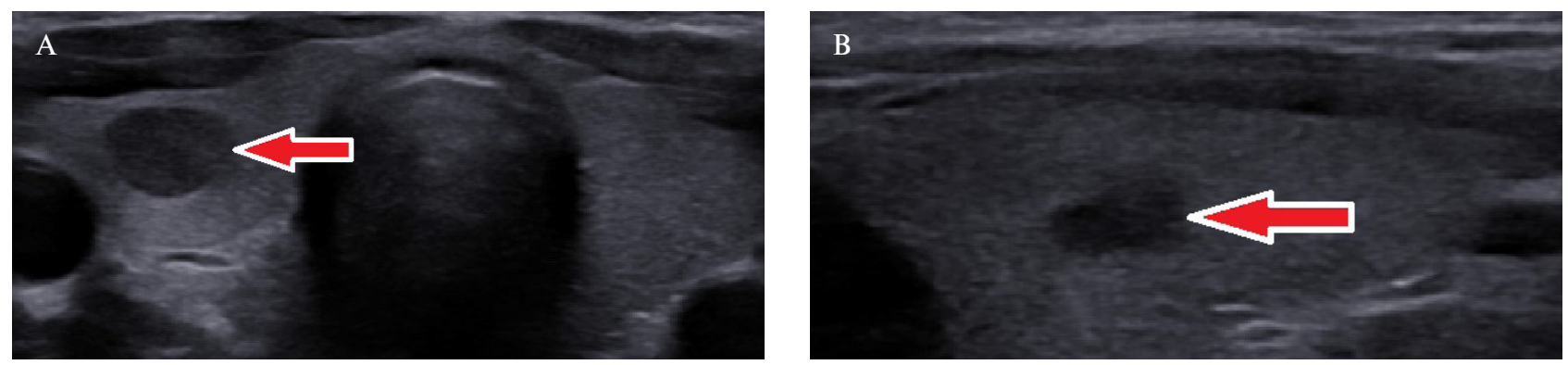

Fig. 4. Patient 2. Ultrasonography of the thyroid gland; hypoechoic $5 \mathrm{~mm}$ thyroid nodule in each lobe without calcifications; confirmed medullary carcinoma (A,B).

\section{DISCUSSION}

We describe the presenting symptoms and signs of MEN2B in two children. Here we report a possible association of GH deficiency as a potential etiology of growth failure and a unique case of cystic ovarian teratoma that occurred in setting of MEN2B. To our knowledge, mature cystic ovarian teratoma representing a novel tumor in MEN2B has not been previously reported in the literature.

A broad phenotypic spectrum has been observed in MEN2B so far. It is critical for pediatricians to maintain a high index of suspicion when evaluating children with any of the clinical signs linked to MEN2B. MTC is a malignant neuroendocrine tumor arising from the parafollicular C-cells of the thyroid gland. It develops within the first years of life in $100 \%$ of MEN2B patients and is the leading cause of death in case of late diagnosis ${ }^{7}$. Early diagnosis is crucial for the optimal care of these patients for better clinical outcome. The problem is that $90 \%$ of patients carry de novo mutations with usually late diagnosis of MEN2B and MTC in childhood or adolescens ${ }^{7,11}$.

The average age of the MEN2B diagnosis is 14.2 years, but MEN2B presents with nonendocrine features early in childhood ${ }^{12}$. A review of the literature revealed that the knowledge of nonendocrine signs of MEN2B would help to lower the age of diagnosis before development of advanced malignant disease, when surgical cure is possible $^{7,13}$. Children diagnosed via associated nonendocrine signs or positive family history had significantly improved overall survival compared to children diagnosed via symptoms of MTC (100\% vs $53.3 \%)\left(\right.$ ref. $\left.^{8}\right)$.

Most studies showed that more than $95 \%$ of patients with MEN2B have M918T mutation in exon 16 of the $R E T$ proto-oncogene $\mathrm{e}^{1-4}$. These observations correlate with genetic analysis in our cases, de novo M918T mutation in the RET proto-oncogene has been identified in both patients.

Brauckhoff et al investigated the early clinical manifestations of 25 patients with MEN2B and reported that less than $20 \%$ of patients had the typical MEN2B features during the first year of life. The earliest features were mainly bowel problems due to diffuse intestinal ganglioneuromatosis (constipation, feeding difficulties in infancy, megacolon) and alacrima. The inability of children with MEN2B to cry tears is an important clinical symptom for early diagnosis preceding the development of MTC. This feature appeared at a mean age of 0.15 years and was present in $91 \%$ of cases ${ }^{6}$. Redlich et al described alacrima in $16.7 \%$ of cases ${ }^{8}$. MEN2B patients can also complain of upper GI symptomatology and esophageal manifestations such as difficulty swallowing and vomiting ${ }^{14,15}$. Brauckhoff et al suggest association within severe gastrointestinal signs and worse MTC aggressiveness and MTC prognosis ${ }^{12}$.

The earliest features in our patients are in agreement with literature data desribed above. Our retrospective analysis confirmed alacrima as the typical early MEN2B feature in both of our patients from birth. According to our patients ' parents, crying with tears have appeared in children approximately from school age. Patient 1 suffered from constipation of unknown etiology and failure to thrive with feeding difficulties from infancy. Importantly, patient P2 had failure to thrive with feeding difficulties, but he didn't suffer from constipation. In our cases, upper GI symptomatology such as difficulty swallowing, heartburn, nausea and vomiting during the childhood were the main clinical symptoms in patient P1 suggested esophageal abnormalities typical for MEN2B.

On the other hand mucosal neuromas and marfanoid habitus may not be clinically apparent until several years of age $^{6,7}$. Retrospective analysis of MEN2B affected children in Germany (GPOH-MET registry; the German Society for Paediatric Oncology and Haematology-Malignant Endocrine Tumours) revealed in $88 \%$ of patients oral and dental findings as the most frequent manifestations (oral neuromas of tongue, lips, buccal mucosa, enlarged, nodular lips, high-arched palate, gingival hyperplasia, prognatic mandible, spacing of anterior teeth), followed by musculoskeletal symptoms in $79 \%$ of patients (marfanoid habitus, short stature, foot or hip deformity, muscle hypotonia, lordosis, kyphosis, scoliosis, slipped femoral capital epiphysis) and gastrointestinal signs in $83 \%$ of patients (constipation, diarrhea, low weight). Slipped femoral capital epiphysis was described in this study in 3 patients $(12.5 \%)$ at a mean age of $13.9\left(\right.$ ref. $\left.^{8}\right)$.

Our patients had marfanoid features with long and thin extremities, facial stigmatization with a thin elongated face, typical thickened lips and mucosal neuromas of the tongue and lips at the time of MEN2B diagnosis, in the second decade of life. It's very interesting that no- 
ticeably enlarged lips without neuromas have been present in our patients from early childhood. This parental observation is in agreement with photo documentation (Fig. 1,3). Both children had tooth malposition with central diastema. Patient 1 suffered from muscle hypotonia, pes cavus causing frequent falls as a toddler and she was operated on for a slipped femoral capital epiphysis on the right side at the age of 13 years.

We report a possible association of growth disorder and GH deficiency with MEN2B. Such potential association has been rarely published in the literature so far. Redlich et al revealed short stature with the growth below the third percentile in 12 of $24(50 \%)$ children with MEN2B and one of these children was treated with the growth hormone. In $40 \%$ of these patients, low levels of IGF-I and IGFBP-3 (insulin-like growth factor-binding protein 3) were detected ${ }^{8}$.

We have the same experience with growth retardation and GH deficiency with MEN2B. Examination for short stature with linear growth below the 3rd percentile without any progressive growth delay, but outside the genetically predicted range related to the parents' heights, led to the diagnosis of MEN2B in our patient P2. We confirmed idiopathic, isolated, partial GH deficiency with low GH level during the GH stimulation tests and low level of IGF-I. The patient's phenotype and our experience with patient P1 led us to the final diagnosis of MEN2B syndrome.

Discordant data exist on pediatric ovarian pathology which may rarely arise in patients affected by MEN syndromes. The only ovarian tumors associated with MEN, less described in the literature, appear to be carcinoids. The majority of these tumors occuring in patients with MEN1 and only 9\% in patients with MEN2A (ref. ${ }^{10,16,17}$ ). It is the first time that we report a MEN2B patient who developed mature cystic ovarian teratoma representing a novel ovarian tumor in this disease. This new clinical spectrum was presented in individuals of Central European descent in the Czech Republic. Mature cystic teratoma is the most common ovarian tumor in children and adolescents ${ }^{18}$. Although several case reports have been published, a case of cystic ovarian teratoma in pediatric MEN2B has not yet been established. We suggest that cystic ovarian teratoma observed in our patient $\mathrm{P} 1$ could be considered as a possible new feature in young girls with MEN2B. PET/CT revealed suspected teratoma of the right ovary in our patient $\mathrm{P} 1$, and histopathological examination of the ovary, which plays a significant role for the proper management showed evidence of cystic ovarian teratoma. One thing which was quite interesting for us was that direct sequencing of exons revealed a genetic change that is de novo mutation (c.2753T>C, p.M918T) of the RET proto-oncogene in both patients $\mathrm{P} 1$ and $\mathrm{P} 2$. Thus, we suggest that our findings add new data on the clinical spectrum and the genotype-phenotype relationships and differences of MEN2B. A previously unreported finding in the context of a rare condition raises the possibility of a link between MEN2B and the occurence of ovarian teratoma. Overall, the evidence in this study suggests that cystic ovarian teratoma should be regarded as other new tumor arising in MEN2B. We suggest that patients with MEN2B need to be followed for the development of these non-familial forms of tumors which arise in subjects with these genetic syndromes. Based on data such as these, there may be no distinct biochemical markers that allow identification of familial versus non-familial forms of the ovarian teratomas in MEN2B.

ATA guidelines recommend in patients with known genetic mutation prophylactic thyroidectomy before the age of 1 year, even in the first months ${ }^{11}$. The problem is that most of MEN2B patients carry de novo mutations. Shankar et al highlights prophylactic thyroidectomy in 9 weeks old infant with familial MEN2B in whom microscopic MTC has been already revealed ${ }^{19}$. An international, multicentre, retrospective study including 338 patients with MEN2B confirmed a significant difference in remission status between patients who underwent thyroidectomy before and after the age of 1 year ${ }^{20}$. On the other hand, Brauckhoff et al showed that MTC was potentially curable if thyroidectomy was performed up to the age of 4 years in a cohort of 41 patients with MEN2B with de novo mutations ${ }^{13}$.

Diagnosis of MTC with de novo M918T mutation in the RET proto-oncogene was late in both of our patients, in the second decade of life, but typical nonendocrine symptoms of MEN2B were present in early childhood. Patient P1 has had advanced MTC at the time of diagnosis with high level of pre and postoperative calcitonine suggesting that the operation wasn't curable and the prognosis is uncertain. In comparison, MTC wasn't advanced in patient $\mathrm{P} 2$ and the operation was curable. His preoperative level of calcitonine was much lower than in patient P1 and postoperative level was in normal range. Our patient's prognosis is favorable. We can speculate on the effect of growth hormone deficiency on the stage of MTC at the time of diagnosis in this patient.

\section{CONCLUSION}

Here, we illustrate clinical manifestations in two cases of the disease linked to the de novo gene mutation M918T in the RET proto-oncogene. These cases remind us that children with MEN2B may develop cystic ovarian teratoma, a novel condition in MEN2B which has never been described in previous publications. Our observation extends the list of known tumors in MEN2B, expands the recognized disease spectrum and highlights the importance of sequencing analyses in MEN2B, and may be crucial for genetic counseling, endocrine care provision and better clinical outcome.

\section{ABBREVIATIONS}

MEN: Multiple endocrine Neoplasia; MTC: Medullary thyroid carcinoma; ATA: American Thyroid Association, GI: Gastrointestinal tract; GH: Growth hormone; BMI: Body mass index; FNAB: Fine needle aspiration biopsy; P: the index patient; MRI: Magnetic resonance imaging; 
PET/CT: Positron emission tomography-computed tomography; IGF-I: Insulin-like growth factor-I; IGFBP-3: Insulin-like growth factor-binding protein 3.

Acknowledgment: We are grateful to the parents of the two reported patients who made contributions to acquisition of data described in the manuscript.

Research funding: Progress Q-39. The project "Modernization and instrumental upgrade of the National Center for Medical Genomics" (reg. No. CZ.02.1.01/0.0 /0.0/18_046/0015515) is supported by the Operational Programme Research, Development and Education. We acknowledge the CF Department of Medical Genetics, Faculty of Medicine in Pilsen, Charles University Prague and the University Hospital in Pilsensupported by the NCMG research infrastructure (LM2018132 funded by MEYS CR) for their support. This work was supported by the Ministry of Health of the Czech Republic AZV (NU21-01-00448) and MHCZ-DRO (Institute of Endocrinology, 00023761) grants.

Authors contributions: All the authors have accepted responsibility for entire content of this submitted manuscript and approved submission.

Conflict of interest statement: The authors state that there are no conflicts of interest regarding the publication of this article.

\section{REFERENCES}

1. Carlson KM, Dou S, Chi D, Scavarda N, Toshima K, Jackson CE, Wells SA Jr, Goodfellow PJ, Donis-Keller H. Single missense mutation in the tyrosine kinase catalytic domain of the RET protooncogene is associated with multiple endocrine neoplasia type 2B. Proc Natl Acad Sci U S A 1994;91:1579-83.

2. Hofstra RMW, Landsvater RM, Ceccherini I, Stulp RP, Stelwagen T, Luo Y, Pasini B, Höppener JW, van Amstel HK, Romeo G. A mutation in the RET proto-oncogene associated with multiple endocrine neoplasia type $2 \mathrm{~B}$ and sporadic medullary thyroid carcinoma. Nature 1994;367:375-6.

3. Gimm O, Marsh DJ, Andrew SD, Frilling A, Dahia PLM, Mulligan LM, Zajac JD, Robinson BG, Eng C. Germline dinucleotide mutation in codon 883 of the RET proto-oncogene in multiple endocrine neoplasia Type 2B without codon 918 mutation. J Clin Endocrinol Metab 1997:82:3902-4.

4. Hansford JR, Mulligan LM. Multiple endocrine neoplasia type 2 and RET: from neoplasia do neurogenesis. Journal of Medical Genetics 2000;37:817-27.

5. Mathiesen JS, Kroustrup JP, Vestergaard P, Madsen M, Stochholm K Poulsen PL, Rasmussen AK, Rasmussen UF, Schytte S, Pedersen HB, Hahn ChH, Bentzen J, Gaustadnes M, Orntoft TF, Hansen TO, Nielsen FC, Brixen K, Frederiksen AL, Godballe $\mathrm{Ch}$. Incidence and prevalence of multiple endocrine neoplasia 2B in Denmark: a nationwide study Endocr Relat Cancer 2017;24(7):L39-L42.
6. Brauckhoff M, Machens A, Hess S, Lorenz K, Gimm O, Brauckhoff K, Sekulla $C$, Dralle H. Premonitory symptoms preceding metastatic medullary thyroid cancer in MEN 2B: an exploratory analysis. Surgery 2008:144:1044-50.

7. Castinetti F, Moley J, Mulligan L, Waguespack SG. A comprehensive review on MEN2B. Endocr Relat Cancer 2018;25:T29-39.

8. Redlich A, Lessel L, Petrou A, Mier P, Vorwerk P. Multiple endocrine neoplasia type 2B: Frequency of physical stigmata-Results of the GPOH-MET registry. Pediatr Blood Cancer 2020;67:e28056.

9. Andrade S, Sirchia F, Faleschini E, Barbi E. A Girl with Delayed Puberty and Bumpy Lips. J Pediatr 2018;203:454-54.

10. Papageorgiou T, Stratakis CA. Ovarian tumors associated with multiple endocrine neoplasias and related syndromes (Carney complex, Peutz-Jeghers syndrome, von Hippel-Lindau disease, Cowden's disease). Int J Gynecol Cancer 2002;12(4):337-47.

11. Wells SA, Asa SL, Dralle H, Elisei R, Evans DB, Gagel RF, Lee N, Machens A, Moley JF, Pacini F, Raue F, Raue KF, Robinson B, Rosenthal MS, Santoro M, Schlumberger M, Shah M, Waguespack SG. Revised American Thyroid Association guidelines for the management of medullary thyroid carcinoma. Thyroid 2015;25:567-610.

12. Brauckhoff $M$, Gimm O, Weiss CL, Ukkat J, Sekulla $C$, Brauckhoff K, Thanh PN, Dralle H. Multiple endocrine neoplasia 2B syndrome due to codon 918 mutation: clinical manifestation and course in early and late onset disease. World J Surg 2004;28(12):1305-311.

13. Brauckhoff M, Machens A, Lorenz K, Bjoro T, Varhaug JE, Dralle H. Surgical curability of medullary thyroid cancer in multiple endocrine neoplasia 2B. Ann Surg 2014;259:800-6.

14. Cohen MS, Phay JE, Albinson C, DeBenedetti MK, Skinner MA, Laimore TC, Doberty GM, Balfe DM, Wells SA Jr, Moley JF. Gastrointestinal manifestations of multiple endocrine neoplasia type 2. Ann Surg 2002;235(5):648-54.

15. Gibbons D, Camilleri M, Nelson AD, Eckert D. Characteristics of chronic megacolon among patients diagnosed with multiple endocrine neoplasia type 2B. United European Gastroenterol J 2016;4(3):44954.

16. Duh QY, Hybarger CP, Geist R, Gamsu G, Goodman PC, Gooding GA, Clark $\mathrm{OH}$. Carcinoids associated with multiple endocrine neoplasia syndromes. Am J Surg 1987;154:142-48.

17. Tamsen A, Mazur MT. Ovarian strumal carcinoid in association with multiple endocrine neoplasia, type IIA. Arch Pathol Lab Med 1992;116:200-3.

18. Lala SV, Strubel N. Ovarian neoplasms of childhood. Pediatr Radiol 2019;49(11):1463-475.

19. Shankar RK, Rutter MJ, Chernausek SD, Samuels PJ, Mo JQ, Rutter MM. Medullary thyroid cancer in a 9-week-old infant with familial MEN 2B: Implications for timing of prophylactic thyroidectomy. Int J Pediatr Endrocrinol 2012;2012(1):25.

20. Castinetti F, Waguespack SG, Machens A, Uchino S, Lazar K, Sanso G, Else T, Dvorakova S, Qi XP, Elisei R, Maia AL, Glod J, Lourenço DM Jr, Valdes N, Mathiesen J, Wohllk N, Bandgar TR, Drui D, Korbonits M, Druce MR, Brain C, Kurzawinski T, Patocs A, Bugalho MJ, Lacroix A, Caron P, Fainstein-Day P, Chazot FB, Klein M, Links TP, Letizia C, Fugazzola L, Chabre O, Canu L, Cohen R, Tabarin A, Uroic AS, Maiter D, Laboureau S, Mian C, Peczkowska M, Sebag F, Brue T, Mirebeau-Prunier D, Leclerc L, Bausch B, Berdelou A, Sukurai A, Vlcek P, Krajewska J, Barontini M, Vaz Ferreira Vargas C, Valerio L, Ceolin L, Akshintala S, Hoff A, Godballe Ch, Jarzab B, Jimenez C, Eng Ch, Imai T, Schlumberger M, Grubbs E, Dralle H, Neumann $\mathrm{HP}$, Baudin E. Natural history, treatment, and long-term follow up of patients with multiple endocrine neoplasia type 2B: an international, multicentre, retrospective study. Lancet Diabetes Endocrinol 2019;7(3):213-20. 